\section{Japan speeds up mission to unravel genetic diseases}

David Cyranoski, Tokyo

A sealed card is at the heart of Japan's bid to unmask the genetics behind various diseases including arthritis and asthma. With the opening of its new facility in Tokyo, RIKEN, the Institute of Physical and Chemical Research, plans to push Japan to the forefront of the global effort to interpret the human genome.

The facility will use its own sealed-card technology to map single-nucleotide polymorphisms (SNPs), the single-base variations that are scattered throughout the genome. SNPs are thought to be behind most genetic variations - from eye colour to disease susceptibility.

RIKEN's technology allows researchers to read up to 384,000 DNA samples per day, using much smaller blood samples than were previously required. The institute hopes the technique will allow it to match and even surpass existing efforts, such as the SNP Consortium of international drug companies and academic laboratories.

"The capacity they are trying to achieve will put them at the top of the heap," says Pui-Yan Kwok, a genetics researcher at Washington University School of Medicine in St Louis, Missouri.

The new facility will be managed by Yusuke Nakamura, who is also head of Tokyo University's Human Genome Center. This centre has already identified about 90,000 new SNPs within the Japanese population, and aims to reach 150,000 by next March.

Many diseases, such as cancer and diabetes, are associated with several genes. SNPs can be used to identify those genes - a SNP located close to a gene associated with a certain disease tends to be inherited with it, and so can act as a marker for the disease.

But some SNPs are found within the disease genes themselves, and it is these that Nakamura is most interested in. This is because they can help identify not just groups of genes, but also particular combinations of alleles — gene sequences with small variations - that are associated with the disease.

Nakamura plans to genotype 120,000 SNPs for each of 768 Japanese patients. This will mean analysing about 100 million SNPs, a mammoth task that he hopes to finish within a year.

"It is necessary to look simultaneously at hundreds of thousands of SNPs in an individual's genome and then compare these with other individuals - thousands or tens of thousands of them," says Nakamura.

RIKEN will achieve this kind of capacity by combining its sealed, 384-well card with a technique for amplifying DNA from blood

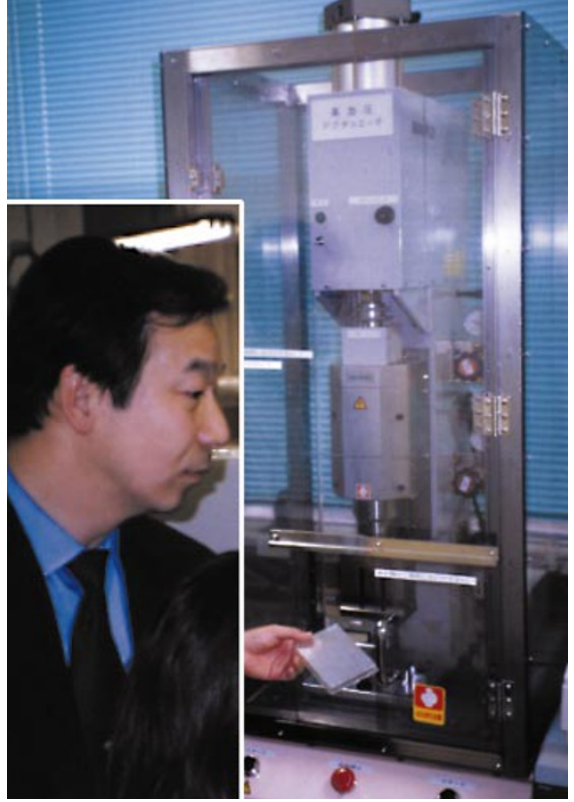

Rapid response: Yusuke Nakamura says the sealed-card technology will speed up analysis.

samples, and an assay that can quickly genotype SNPs. Together these reduce the size of the sample required, reducing costs to "less than one tenth of the previous method", says Nakamura.

The sealed card helps to speed up the entire process, explains Nakamura. Conventional cards have to be processed one by one - placed in a scanning machine that incubates the materials in the wells and catalyses the necessary reaction for detection. But RIKEN's cards can be dropped into hot water in large batches, allowing 1,000 cards to be dealt with each day.

As RIKEN ramps up its identification of SNPS, others are not sitting idle. The Whitehead Institute for Biomedical Research at the Massachusetts Institute of Technology is mounting an effort of similar size and scope to that at RIKEN. This will focus on American and European populations, says Eric Lander, director of the institute's Center for Genome Research. "I reckon that these will be the largest efforts in the world — and quite complementary," he says.

Nakamura says he has already found SNPs related to arthritis and asthma, and expects to find many more as his genotyping project develops. Like the SNP Consortium, RIKEN plans to make all of its findings freely available on the Internet. It will post the first 10,000 of the genotyped SNPs by May or June of this year, and put all of the genotyped SNPs information in a public database next year.

http://snp.ims.u-tokyo.ac.jp http://snp.cshl.org

\section{South Africa may keep door closed to generic AIDS drugs}

\section{Michael Cherry, Cape Town}

Drug companies expect the South African government to hold off from importing cheap generic copies of their AIDS drugs following the withdrawal of their lawsuit against the government last week.

Although the 39 drug-makers withdrew their lawsuit unconditionally, officials of South Africa's Pharmaceutical Manufacturers Association say the government has privately undertaken to import only branded versions of drugs already under patent in South Africa.

The government's only public promise is to consult the drug companies when drawing up regulations relating to the section of the 1997 Medicines and Related Substances Control Amendment Act to which the companies objected in their lawsuit.

The government had previously said it would use either parallel imports or compulsory licensing — allowing domestic production of a patented drug to obtain drugs at lower prices than offered by patent holders. It has not committed itself to withdrawing that intention.

Earlier last week, South Africa's Medicines Control Council announced that it had registered the anti-AIDS drug nevirapine for use in preventing the mother-to-child transmission of HIV. The drug's manufacturer, Boehringer Ingelheim, has agreed that any possible resistance problems can be monitored. The decision opens the way - in theory - for the drug to become available on prescription.

But AIDS activists' euphoria over the lawsuit might be short-lived. At a press conference following the decision, health minister Manto Tshabalala-Msimang said hopes should not be raised that anti-AIDS drugs would become available. She said their widespread use would be hampered by a lack of adequate infrastructure, high costs and drug resistance.

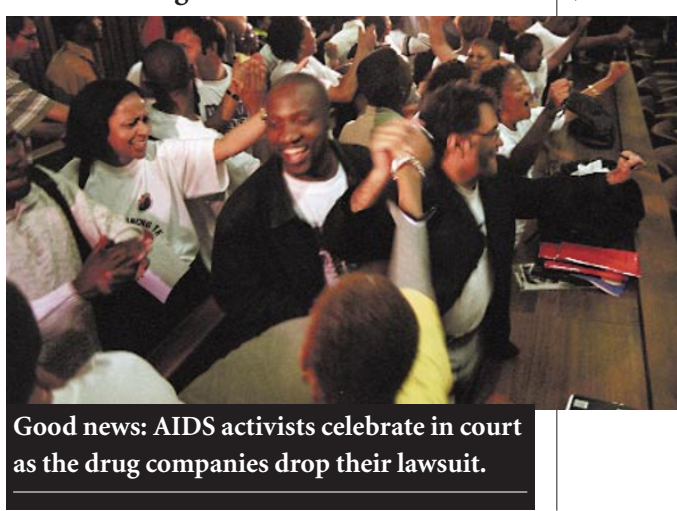

